\title{
Article \\ Exploring Factors That Influence Injured Patients' Outcomes following Road Traffic Crashes: A Multi-Site Feasibility Study
}

\author{
Rayan Jafnan Alharbi ${ }^{1,2,3, *(\mathbb{D})}$, Virginia Lewis ${ }^{4}\left(\mathbb{D}\right.$, Omar Othman ${ }^{5}$ and Charne Miller ${ }^{1,6}$ \\ 1 School of Nursing \& Midwifery, La Trobe University, Melbourne, VIC 3083, Australia; \\ charne.miller@unimelb.edu.au \\ 2 Department of Emergency Medical Service, Jazan University, Jazan 45142, Saudi Arabia \\ 3 Cabrini Research \& Education, Cabrini Health, Melbourne, VIC 3144, Australia \\ 4 Australian Institute for Primary Care and Ageing, La Trobe University, Melbourne, VIC 3083, Australia; \\ v.lewis@latrobe.edu.au \\ 5 King Saud Medical City, Ministry of Health, Riyadh 12746, Saudi Arabia; o.othman@ksmc.med.sa \\ 6 Department of Nursing, Faculty of Medicine, Dentistry \& Health Sciences, The University of Melbourne, \\ Melbourne, VIC 3010, Australia \\ * Correspondence: ralharbi@windowslive.com or r.alharbi@latrobe.edu.au; Tel.: +61-3-9479-1798
}

check for updates

Citation: Alharbi, R.J.; Lewis, V.; Othman, O.; Miller, C. Exploring Factors That Influence Injured Patients' Outcomes following Road Traffic Crashes: A Multi-Site

Feasibility Study. Trauma Care 2022, 2, 35-50. https://doi.org/10.3390/ traumacare 2010004

Received: 27 January 2022

Accepted: 21 February 2022

Published: 23 February 2022

Publisher's Note: MDPI stays neutral with regard to jurisdictional claims in published maps and institutional affiliations.

Copyright: (C) 2022 by the authors. Licensee MDPI, Basel, Switzerland. This article is an open access article distributed under the terms and conditions of the Creative Commons Attribution (CC BY) license (https:// creativecommons.org/licenses/by/ $4.0 /)$.

\begin{abstract}
Background: Injuries arising from Road Traffic Crashes (RTCs) are a major health problem in Saudi Arabia (SA). The purpose of the study was to determine the feasibility of conducting a multicenter research study to explore factors that influence the mortality of RTC-related trauma patients in SA. Methods: A multi-center observational study was undertaken involving both prospective and retrospective data collected from three hospitals. In-hospital patient mortality thirty days post-crash was the primary outcome variable. The feasibility of the study methods including the quality of data were evaluated and pilot results pertaining to factors predicting mortality were examined. Results: The overall mortality rate ( $n=572$ RTC victims) was (7.5\%). A logistic regression model identified four independent predictors of mortality following an RTC: treatment at a non-trauma center-based hospital, SBP $\leq 90 \mathrm{mmHg}$, GCS $\leq 8$, and ISS $\geq 20$. With respect to the assessment of the study method's feasibility, missing data was problematic, especially for variables pertaining to crash characteristics and prehospital care. Conclusions: Collecting multi-center injury data in SA has logistic challenges, predominantly associated with the comparability and completeness of data sets as well as the need for manual screening and data collection at some institutions. Despite these limitations, this study has demonstrated the feasibility of a method that could be utilized in further large nationwide studies to understand and examine the factors that influence injured patients outcomes following RTCs.
\end{abstract}

Keywords: traffic crash; traumatic injury; prehospital care; mortality

\section{Introduction}

Injuries arising from Road Traffic Crashes (RTCs) are a major health problem globally [1], with RTCs being the most common mechanism of injury-related deaths in Lowand Middle-Income Countries (LMICs) [2]. Among those aged 15-29 years, RTC injuries are the leading cause of death globally [3]. The burden of RTC injury affects all countries [4]; however, the burden is exponentially greater in LMICs, where $90 \%$ of the world's RTCs occur $[5,6]$. The RTC burden in LMIC countries is illustrated by Saudi Arabia (SA) where RTC injuries are the second leading cause of death [7], with 6025 deaths and 30,217 injured individuals reported between September 2017 and September 2018 [8]. Efforts to reduce the burden of RTC-related mortality and morbidity are required. This study examined the feasibility of conducting research in SA to explore factors that influence the mortality of RTC-related trauma patients. 
The current SA trauma system varies by region and expertise with respect to trauma care. For example, major trauma centers only exist in a few cities, only two large hospitals have a registry, and prehospital advanced life support (ALS) care is not available in all regions [9]. Prehospital care is defined as a medical subspecialty that focuses on caring for ill or injured patients on-scene and during transport to the hospital [10]. A previous study in Saudi Arabia found that a range of crash characteristics were associated with an increased risk of fatal crashes, such as lighting conditions, the number of vehicles involved, and the direction of the collision [11]. However, there is limited literature examining other risk factors; for example, driver factors including speed and fatigue, and prehospital and hospital risk factors such as trauma team activation and hospital level of care.

The maturity of a national or regional trauma system designed to provide multispecialty care for the injured has been observed to impact patient outcomes [12]. The lack of a national trauma system presents a challenge to understanding patient characteristics and outcomes following traumatic injury events. A review of the SA trauma system undertaken in 2020 identified some gaps in the system [9]. The prehospital response to RTCs was noted as an important area where further research was needed. For example, the study observed an absence of prehospital guidelines either for field triage or trauma destination protocols such as trauma bypass. Further, the role of tertiary hospitals in the care of traumatic injury patients was unclear, with no guidelines for referral between the hospitals identified [9].

As traumatic injuries are a leading cause of death in SA, research into trauma care is justified to enhance the health, safety, and wellbeing of the population [9]. Further, an understanding of the factors associated with road traffic mortality will help to identify areas where potential efforts can be directed to strengthen the trauma care system, explore the prevention of death, and improve trauma patient outcomes in SA. The primary purpose of the study was to determine whether it is feasible to source a comprehensive multi-center dataset in SA. The secondary purpose of the study was to analyse data from participating hospitals, dependent on availability, to identify factors influencing the mortality of RTCrelated trauma patients in SA.

\section{Materials and Methods}

\subsection{Study Design}

This study was conducted in accordance with an a priori published protocol [13]. An observational study was conducted that involved both retrospective and prospective data from three purposefully selected hospitals with different trauma services in different geographical locations and cities in SA. This study was approved by the Institutional Review Board Committee at King Saud Medical City, SA (H-01-R-053), the General Department of Research and Studies at the SA Ministry of Health: Buraidah Central Hospital (1440-1249939), Yanbu General Hospital (1440-1398648), and the La Trobe University Human Research Ethics Committee (HEC19095).

Site one was the King Saud Medical City (KSMC), located in the capital city of SA (Riyadh city), where one quarter of the country's population resides (8 million) [14]. The Riyadh region has 47 Ministry of Health $(\mathrm{MOH})$ hospitals [9], of which KSMC is the largest in the region, with a bed capacity of 1400 . KSMC receives most of the emergency cases, including trauma cases, in the region. The KSMC has a trauma registry known as the Saudi Trauma Registry (STAR) and is in the process of converting the hospital to a level 1 trauma center [15]. Site two was the Buraidah Central Hospital (BCH), located in the middle of the country in the Qassim region, where approximately 1.4 million people live. $\mathrm{BCH}$ is in the capital city of Qassim Buraidah. There are $18 \mathrm{MOH}$ hospitals in Qassim [9]; $\mathrm{BCH}$ is the largest, with a 400-bed capacity. The majority of trauma cases in Qassim are transported/transferred to the $\mathrm{BCH}$, which has the highest level of tertiary care in the region for injured patients. However, the $\mathrm{BCH}$ does not have a trauma registry and the hospital is not classified as a trauma center. Site three was the Yanbu General Hospital (YGH), located in Yanbu city, where 300,000 people reside. YGH has a capacity of approximately 300 beds 
and is considered a main but non-specialist hospital in the city. Similar to $\mathrm{BCH}, \mathrm{YGH}$ does not have a trauma registry and trauma care resources are limited in the hospital.

All sites provided deidentified data from hospital administrative records for the study where available. Information was extracted from routinely collected prehospital and hospital data collection. In addition, the two hospitals without a trauma registry $(\mathrm{BCH}$ and YGH) collected data from patients who met the study eligibility criteria and from whom consent was provided to gather the data. A consent form in two languages (Arabic and English) was used by local research partners at $\mathrm{BCH}$ and $\mathrm{YGH}$ when collecting additional data. No patient consent was required for the KSMC site as only deidentified data from the KSMC trauma registry was used. More details regarding the recruitment and consenting process are available in the published protocol [13].

\subsection{Patients and Data Collection}

All adult (aged 18 years or more) patients involved in a road crash-related injury and admitted to one of the three selected hospitals for the period from May to July 2019 were included. Patients aged under 18 years and/or those deemed dead on arrival at the hospital were excluded. The RTCs involved motor vehicles, motorcycles, pedal cycles, pedestrians, and other transport-related circumstances such as bus crashes, heavy vehicles, and lorries. All patients who met the eligibility criteria were included regardless of their Injury Severity Score (ISS). The ISS is commonly used to assess trauma patients' injury severity, with higher scores indicating a more significant injury.

The primary outcome variable was thirty-day, in-hospital mortality post-crash. In April 2018, KSMC implemented a trauma registry [15], collecting data on trauma patients' sociodemographic variables (such as age and gender), crash characteristics (such as mechanism of injury), physiological assessment (such as blood pressure and pulse), and prehospital and hospital variables (such as mode of arrival, ICU admission, and hospital length of stay). As such, retrospective data on eligible patients attending KSMC for the study period were requested from the STAR. Data were provided to the research team as a digital file. At BCH and YGH, a local research partner (nurses/health professionals working within the hospital) collected prospective data from consenting patients at the two hospitals for the same period using a customized data collection tool. Data were captured prospectively, through patient interview as well as from information recorded in the hospitals. Existing healthcare records were the primary source of information about patients. The data collection tool was developed to accommodate the pre-existing fields in the institutions' medical records to optimize data capture. As such, the data collected at these two hospitals did not include nor take the exact form of all the data fields in the trauma registry's minimum data set.

Data were received as hard copies from the $\mathrm{BCH}$ and $\mathrm{YGH}$ and were manually entered. Where there were differences in the way variables were recorded, a new variable was created and the capacity to reconcile the fields was examined as part of the feasibility assessment. An independent researcher completed a 10\% validation of randomly selected cases, demonstrating $95 \%$ accuracy of data entry.

\subsection{Statistical Analyses}

Descriptive data regarding patients' sociodemographic variables, crash characteristics, physiological assessment, and prehospital and hospital variables were collected. Thirty-day, in-hospital mortality was examined. To identify possible mortality risk factors, bivariate analyses were performed where sufficient data were available to permit investigation. Based on previous literature [16], continuous variables were dichotomized for the purpose of the analysis using clinically relevant cut-off points, including Glasgow Coma Scale (GCS $\leq 8$ vs. $>8$ ), ISS (<20 vs. $\geq 20$ ) and systolic blood pressure (SBP $\leq 90 \mathrm{mmHg}$ vs. $>90 \mathrm{mmHg}$ ). The GCS measures a patient's level of consciousness, with lower scores indicating a low level of consciousness. The SBP measures the pressure that blood force exerts on the walls of arteries each time it beats. 
Further, the following variables were recategorized to create consistent binary categories that align with prior publications: age (18-54 vs. $\geq 55$ ); mechanism of injury (motor vehicle driver vs. all other); hospital arrival mode (ambulance vs. non-ambulance); and hospital level (trauma center vs. non-trauma center) $[17,18]$. The dependent variable (patient 30-day, in-hospital outcome) was classified as died and survived. In this study, people who were discharged home were assumed to have survived.

Given that Fisher's exact test is more accurate in analyzing small samples [19], in this study, the unadjusted $p$ values were derived from chi-square tests or Fisher's exact tests. Based on previous research [20-22], any variables with a difference in outcome that had a $p$ value of $<0.02$ were included in the logistic regression analysis. In addition to the above criteria, we included only variables where data were available from all hospitals (e.g., GCS, SBP, head injury) in the logistic regression model. After these potential predictors were identified, a logistic regression analysis was performed to identify independent risk factors for RTC mortality in the population and to estimate adjusted and unadjusted odds ratio (OR) and 95\% confidence intervals (CI). All analyses were performed using Statistical Package for Social Sciences version 26.0 (SPSS Inc., Chicago, IL, USA). An independent review of the statistics plan and statistical analyses was performed in consultation with a statistician. The statistical assumptions of a regression analysis were checked.

\section{Results}

A total of 572 trauma patients were included in this study from the three study sites. Table 1 presents a summary of patient characteristics by study site. Of the total study sample, $88.6 \%$ and $11.4 \%$ were male and female, respectively. The average age of patients was 33.3 years ( $\mathrm{SD}=13.4)$. Of the 357 patients (from the two prospective sites, $\mathrm{BCH}$ and YGH), $59.6 \%$ of patients were married, $67.2 \%$ were employed, and $67.6 \%$ had tertiary education. The average weight of patients was $72.5 \mathrm{~kg}(\mathrm{SD}=10.3)$.

With regards to the crash characteristics (Table 2), the majority of patients were motor vehicle drivers $(58.2 \%)$ or motor vehicle passengers (24.9\%). Head-on collisions were the most frequently reported type of collision ( $34.8 \%)$. Patients typically arrived at the hospital by ambulance $(79.7 \%)$. Trauma team activation occurred in only about one-fifth (20.7\%) of cases. Mean prehospital physiological assessments were SBP 133.6 (SD = 23.2), HR 97.4 ( $\mathrm{SD}=18.6)$, and RR $20.0(\mathrm{SD}=8.4)$, whereas mean in-hospital physiological assessments were SBP 126.1 (SD = 23.7), HR 90.9 (SD = 19.5), and RR 20.0 (SD = 7.1). The majority of the crashes (56.3\%) occurred during AM hours (12 am-12 pm). Of the total population, 444 patients $(79.7 \%)$ arrived by ambulance, whereas 106 patients $(19.0 \%)$ arrived by private vehicles. The majority of patients presented with minor injury $64.4 \%$, ISS $<13$ followed by severe injury $11.6 \%$, ISS $=16-19$. Head injuries were the most common injury, present in 165 cases $(28.8 \%)$, followed by injuries to the thorax and the abdomen 122 cases $(21.3 \%)$. Table 3 presents a summary of prehospital and hospital variables.

The overall average number of days in an intensive care unit (ICU) was $0.9(\mathrm{SD}=4.2)$ and average length of hospital stay was 7.0 days $(\mathrm{SD}=10.4)$. Of the total study sample, 488 patients $(85.3 \%)$ were discharged home within the first 30 days after the crash (Table 4). Of 357 patients, $11.4 \%(n=41)$ patients from the two prospective sites $(\mathrm{BCH}$ and $\mathrm{YGH})$ did not agree to provide additional information. It was not possible to compare patients $(n=41)$ who did not consent to provide extra information with those who did, as the data provided by the hospitals did not include the information in the deidentified data. However, it is estimated that those patients were more likely to have a severe injury, and that their next of kin were not available for consent.

In-hospital mortality was 7.5\% $(n=43)$, with similar rates for males and females $(7.5 \%$ and $9.5 \%$, respectively, unadjusted $\mathrm{OR}=0.77,95 \% \mathrm{CI}$ : $0.31-1.91, p=0.57)$. Age was not significantly associated with mortality; mortality among people aged $18-54$ years was $(7.4 \%)$ and among those 55 years or older was (9.6\%) (unadjusted OR $=0.74,95 \%$ CI: $0.28-1.98, p=0.55$ ). However, patients in the older age group were more likely to sustain critical injuries (ISS $\geq 20$ ) compared to the younger group, $23.5 \%$ and $13.8 \%$, respectively, 
(unadjusted OR $=0.51,95 \%$ CI: $0.25-1.03, p=0.06$ ). Similar proportions of patients in the non-trauma-based system hospitals and the trauma-based system hospital (15.9\% and $16.2 \%$, respectively) had high injury severity scores (ISS $\geq 20$ ).

With respect to the assessment of the feasibility of gathering the required data to undertake research about RTC in SA, missing data were evident for some variables and differed across sites. Most of the missing variables were related to crash characteristics and prehospital care. For some variables from the prospective sites, particularly YGH, more than $30 \%$ of the data was missing (e.g., weight, use of seat belt/helmet protection). The amount of missing data is reported in Tables 1-4. Although some of data at the $\mathrm{BCH}$ and YGH sites could be missing because patients did not agree to participate in the study, it is essential information for routine data collection. Variables that were missing that should be part of the hospitals' routinely collected health data include weight, level of education, collision type, seat belt/helmet protection, time to the hospital, and whether any prehospital procedure was performed.

Table 1. Characteristics of the study population.

\begin{tabular}{|c|c|c|c|c|}
\hline Population Descriptor & KSMC & ВCH & YGH & Total \\
\hline Total study participants N (\%) & $215(37.8)$ & $256(44.6)$ & $101(17.6)$ & $572(100)$ \\
\hline \multicolumn{5}{|l|}{ Age (in years) } \\
\hline Mean $( \pm S D)$ & $34.6(13.1)$ & $30.9(13.2)$ & $36.3(14.0)$ & $33.3(13.4)$ \\
\hline Range & $18-76$ & $18-83$ & $18-90$ & $18-90$ \\
\hline \multicolumn{5}{|l|}{ Age group (in years) N (\%) } \\
\hline $18-34$ & $133(61.9)$ & $178(70.1)$ & $54(54.0)$ & $365(64.1)$ \\
\hline $35-54$ & $56(26.0)$ & $59(23.2)$ & $37(37.0)$ & $152(26.7)$ \\
\hline$\geq 55$ & $26(12.1)$ & $17(6.7)$ & $9(9.0)$ & $52(9.1)$ \\
\hline $\begin{array}{c}\text { Unknown or not documented } \mathrm{N} \\
\text { Gender } \mathrm{N}(\%)\end{array}$ & 0 & 2 & 1 & 3 \\
\hline Male & $192(89.3)$ & $209(87.4)$ & $90(90.0)$ & $491(88.6)$ \\
\hline Female & $23(10.7)$ & $30(12.6)$ & $10(10.0)$ & $63(11.4)$ \\
\hline \multicolumn{5}{|l|}{ Marital status N (\%) } \\
\hline Married & $\mathrm{N} / \mathrm{A}$ & $141(58.5)$ & $63(62.4)$ & $204(59.6)$ \\
\hline Unmarried & $\mathrm{N} / \mathrm{A}$ & $100(41.5)$ & $38(37.6)$ & $138(40.4)$ \\
\hline $\begin{array}{l}\text { Unknown or not documented N } \\
\text { Weight (in kilograms) }\end{array}$ & \multicolumn{3}{|c|}{ Weight (in kilograms) } & 15 \\
\hline Mean $( \pm S D)$ & $\mathrm{N} / \mathrm{A}$ & $72.7(10.1)$ & $64.2(12.4)$ & $72.5(10.3)$ \\
\hline & Height (in centimetres) & 19 & 93 & 112 \\
\hline Mean $( \pm S D)$ & $\mathrm{N} / \mathrm{A}$ & $163.3(18.6)$ & $\mathrm{N} / \mathrm{A}$ & $163.3(18.6)$ \\
\hline \multicolumn{5}{|l|}{ Employment status N (\%) } \\
\hline Employed & $\mathrm{N} / \mathrm{A}$ & $152(64.7)$ & $73(73.0)$ & $225(67.2)$ \\
\hline Unemployed & $\mathrm{N} / \mathrm{A}$ & $83(35.3)$ & $27(27.0)$ & $110(32.8)$ \\
\hline \multicolumn{4}{|l|}{ Education N $(\%)$} & 22 \\
\hline Primary & $\mathrm{N} / \mathrm{A}$ & $4(1.6)$ & 0 & $4(1.6)$ \\
\hline Secondary & $\mathrm{N} / \mathrm{A}$ & $75(30.7)$ & 0 & $75(30.7)$ \\
\hline Tertiary & $\mathrm{N} / \mathrm{A}$ & $165(67.6)$ & 0 & $165(67.6)$ \\
\hline Unknown or not documented $\mathrm{N}$ & $\mathrm{N} / \mathrm{A}$ & 12 & 101 & 113 \\
\hline Time held a driver license (in years) & & & & \\
\hline Mean $( \pm \mathrm{SD})$ & $\mathrm{N} / \mathrm{A}$ & $9.2(10.1)$ & $\mathrm{N} / \mathrm{A}$ & $9.2(10.1)$ \\
\hline Unknown or not documented $\mathrm{N}$ & $\mathrm{N} / \mathrm{A}$ & 155 & 101 & 256 \\
\hline
\end{tabular}

Note: KSMC = King Saudi Medical City; BCH = Buraydah Central Hospital; YGH = Yanbu General Hospital; $\mathrm{N} / \mathrm{A}=$ Not asked or not available; $\mathrm{SD}=$ Standard Deviation . 
Table 2. Crash characteristics.

\begin{tabular}{|c|c|c|c|c|}
\hline Population Descriptor & KSMC & BCH & YGH & Total \\
\hline \multicolumn{5}{|l|}{ Mechanism of injury N (\%) } \\
\hline Motor Vehicle-Driver & $117(54.4)$ & $143(56.3)$ & $72(71.3)$ & $332(58.2)$ \\
\hline Motor Vehicle-Passenger & $42(19.5)$ & 0 & - & $45(7.9)$ \\
\hline Motor Vehicle-Front Passenger & $\mathrm{N} / \mathrm{A}$ & $79(31.1)$ & $11(10.9)$ & $90(15.8)$ \\
\hline Motor Vehicle-Rear Passenger & $\mathrm{N} / \mathrm{A}$ & $5(2.0)$ & $2(2.0)$ & $7(1.2)$ \\
\hline Motorcycle-Driver & $14(6.5)$ & $8(3.1)$ & $13(12.9)$ & $35(6.1)$ \\
\hline Motorcycle-Passenger & $2(0.9)$ & $1(0.4)$ & - & $3(0.5)$ \\
\hline Pedestrian & $1(0.5)$ & $13(5.1)$ & $3(3.0)$ & $17(3.0)$ \\
\hline Pedal cyclist-rider or passenger & $2(0.9)$ & $2(0.8)$ & 0 & $4(0.7)$ \\
\hline Other transport-related circumstance & $37(17.2)$ & 0 & 0 & $37(6.5)$ \\
\hline \multicolumn{5}{|l|}{ Driver liability N (\%) } \\
\hline \multicolumn{5}{|l|}{ Speed } \\
\hline Yes & $\mathrm{N} / \mathrm{A}$ & $217(93.1)$ & 0 & $217(93.1)$ \\
\hline No & $\mathrm{N} / \mathrm{A}$ & $16(6.9)$ & 0 & $16(6.9)$ \\
\hline \multicolumn{5}{|l|}{ Drink driving } \\
\hline Yes & $\mathrm{N} / \mathrm{A}$ & $3(2.0)$ & 0 & $3(2.0)$ \\
\hline No & $\mathrm{N} / \mathrm{A}$ & $144(98.0)$ & 0 & $144(98.0)$ \\
\hline \multicolumn{5}{|l|}{ Fatigue related } \\
\hline Yes & $\mathrm{N} / \mathrm{A}$ & $93(85.3)$ & 1 & $94(85.5)$ \\
\hline No & $\mathrm{N} / \mathrm{A}$ & $16(14.7)$ & 0 & $16(14.5)$ \\
\hline \multicolumn{5}{|l|}{ Distracted/inattentive } \\
\hline Yes & $\mathrm{N} / \mathrm{A}$ & $125(75.8)$ & 0 & $125(75.8)$ \\
\hline No & $\mathrm{N} / \mathrm{A}$ & $40(24.2)$ & 0 & $40(24.2)$ \\
\hline \multicolumn{5}{|l|}{ Collision type $\mathrm{N}(\%)$} \\
\hline Head-on & $\mathrm{N} / \mathrm{A}$ & $56(34.8)$ & 0 & $56(34.8)$ \\
\hline Rear-end & $\mathrm{N} / \mathrm{A}$ & $7(4.3)$ & 0 & $7(4.3)$ \\
\hline Side & $\mathrm{N} / \mathrm{A}$ & $35(21.7)$ & 0 & $35(21.7)$ \\
\hline Roll-over & $\mathrm{N} / \mathrm{A}$ & $29(18.0)$ & 0 & $29(18.0)$ \\
\hline Multiple & $\mathrm{N} / \mathrm{A}$ & $34(21.1)$ & 0 & $34(21.1)$ \\
\hline $\begin{array}{c}\text { Unknown or not documented N } \\
\text { Number of occupants }\end{array}$ & $\mathrm{N} / \mathrm{A}$ & 95 & 101 & 196 \\
\hline Mean $( \pm S D)$ & $\mathrm{N} / \mathrm{A}$ & $2.0(6.3)$ & 0 & $2.0(6.3)$ \\
\hline \multicolumn{5}{|l|}{ Counterpart N $(\%)$} \\
\hline Animal & $1(0.2)$ & $\mathrm{N} / \mathrm{A}$ & $\mathrm{N} / \mathrm{A}$ & $1(0.2)$ \\
\hline Bus $\geq 10$-seater, heavy cargo truck & $4(1.9)$ & $\mathrm{N} / \mathrm{A}$ & $\mathrm{N} / \mathrm{A}$ & $4(1.9)$ \\
\hline Car & $60(27.9)$ & $\mathrm{N} / \mathrm{A}$ & $\mathrm{N} / \mathrm{A}$ & $60(27.9)$ \\
\hline Cycle & $1(0.5)$ & $\mathrm{N} / \mathrm{A}$ & $\mathrm{N} / \mathrm{A}$ & $1(0.5)$ \\
\hline Fixed or stationary object & $11(5.1)$ & $\mathrm{N} / \mathrm{A}$ & $\mathrm{N} / \mathrm{A}$ & $11(5.1)$ \\
\hline Minibus $<10$-seater, pick up tuck, van & $1(0.5)$ & $\mathrm{N} / \mathrm{A}$ & $\mathrm{N} / \mathrm{A}$ & $1(0.5)$ \\
\hline Motorized two-wheeler & $1(0.5)$ & $\mathrm{N} / \mathrm{A}$ & $\mathrm{N} / \mathrm{A}$ & $1(0.5)$ \\
\hline Pedestrian & $8(3.7)$ & $\mathrm{N} / \mathrm{A}$ & $\mathrm{N} / \mathrm{A}$ & $8(3.7)$ \\
\hline $\begin{array}{c}\text { Unknown or not documented } \mathrm{N} \\
\text { Airbag protection } \mathrm{N}(\%)\end{array}$ & $128(59.5)$ & $\mathrm{N} / \mathrm{A}$ & $\mathrm{N} / \mathrm{A}$ & $128(59.5)$ \\
\hline Yes & $\mathrm{N} / \mathrm{A}$ & $64(26.8)$ & $1(1.0)$ & $65(27.7)$ \\
\hline No & $\mathrm{N} / \mathrm{A}$ & $175(73.2)$ & 0 & $175(72.9)$ \\
\hline Unknown or not documented $\mathrm{N}$ & $\mathrm{N} / \mathrm{A}$ & 17 & 100 & 117 \\
\hline \multicolumn{5}{|l|}{ Seat belt/helmet protection $\mathrm{N}(\%)$} \\
\hline Yes & $\mathrm{N} / \mathrm{A}$ & $22(8.8)$ & 0 & $22(8.8)$ \\
\hline No & $\mathrm{N} / \mathrm{A}$ & $228(91.2)$ & 0 & $228(91.2)$ \\
\hline Unknown or not documented $\mathrm{N}$ & $\mathrm{N} / \mathrm{A}$ & 4 & 101 & 105 \\
\hline
\end{tabular}

Note: KSMC = King Saudi Medical City; BCH = Buraydah Central Hospital; YGH = Yanbu General Hospital $\mathrm{N} / \mathrm{A}=$ Not asked or not available; $\mathrm{SD}=$ Standard Deviation . 
Table 3. Prehospital and hospital variables.

\begin{tabular}{|c|c|c|c|c|}
\hline Population Descriptor & KSMC & BCH & YGH & Total \\
\hline \multicolumn{5}{|l|}{$\begin{array}{l}\text { Prehospital physiological } \\
\text { assessment Mean }( \pm \mathrm{SD})\end{array}$} \\
\hline First SBP mmHg & $123.4(22.6)$ & $136.6(21.0)$ & $134.3(28.8)$ & $133.6(23.2)$ \\
\hline Unknown or not documented N & 155 & 64 & 52 & 271 \\
\hline First pulse at scene per min & $95.2(18.5)$ & $\mathrm{N} / \mathrm{A}$ & $100.2(18.6)$ & $97.4(18.6)$ \\
\hline Unknown or not documented $\mathrm{N}$ & 149 & $\mathrm{~N} / \mathrm{A}$ & 52 & 201 \\
\hline First respiratory rate per min & $19.0(10.6)$ & $\mathrm{N} / \mathrm{A}$ & $21.2(4.3)$ & $20.0(8.4)$ \\
\hline Unknown or not documented $\mathrm{N}$ & 154 & $\mathrm{~N} / \mathrm{A}$ & 52 & 206 \\
\hline \multicolumn{5}{|l|}{$\begin{array}{l}\text { Prehospital EMS personnel } \\
\text { involved in care N }(\%)\end{array}$} \\
\hline Yes & $\mathrm{N} / \mathrm{A}$ & $9(4.4)$ & $5(6.7)$ & $14(5.0)$ \\
\hline No & $\mathrm{N} / \mathrm{A}$ & $195(95.6)$ & $70(93.3)$ & $265(95)$ \\
\hline \multicolumn{5}{|l|}{ Paramedic } \\
\hline Yes & $\mathrm{N} / \mathrm{A}$ & $179(87.7)$ & $1(1.3)$ & $180(64.5)$ \\
\hline No & $\mathrm{N} / \mathrm{A}$ & $25(12.3)$ & $74(98.7)$ & $99(35.5)$ \\
\hline \multicolumn{5}{|l|}{ EMT } \\
\hline Yes & $\mathrm{N} / \mathrm{A}$ & $86(42.2)$ & $72(96.0)$ & $158(56.6)$ \\
\hline No & $\mathrm{N} / \mathrm{A}$ & $118(57.8)$ & $3(4.0)$ & $121(43.4)$ \\
\hline \multicolumn{2}{|l|}{ Injury Time N (\%) } & 1 & 0 & 1 \\
\hline $\mathrm{AM}$ & $168(78.1)$ & $99(40.1)$ & $50(49.5)$ & $317(56.3)$ \\
\hline PM & 47 (21.9) & $148(59.9)$ & $51(50.5)$ & $246(43.7)$ \\
\hline \multicolumn{5}{|l|}{ KM from scene to hospital } \\
\hline Mean $( \pm \mathrm{SD})$ & $\mathrm{N} / \mathrm{A}$ & $26.3(16.0)$ & 0 & $26.3(16.0)$ \\
\hline $\begin{array}{l}\text { Unknown or not documented N } \\
\text { Time to hospital (HH:MM) }\end{array}$ & $\mathrm{N} / \mathrm{A}$ & 11 & 101 & 112 \\
\hline Mean $( \pm$ SD) & $\mathrm{N} / \mathrm{A}$ & $00: 28(0: 17)$ & 0 & $00: 28(0: 17)$ \\
\hline Unknown or not documented $\mathrm{N}$ & $\mathrm{N} / \mathrm{A}$ & 12 & 101 & 113 \\
\hline \multicolumn{5}{|l|}{ Prehospital procedure performed } \\
\hline Yes & $29(49.2)$ & $8(3.8)$ & $2(20.7)$ & $39(11.4)$ \\
\hline No & $30(50.8)$ & $201(96.2)$ & $73(97.3)$ & $304(88.6)$ \\
\hline $\begin{array}{l}\text { Unknown or not documented } \mathrm{N} \\
\text { Transportation mode } \mathrm{N}(\%)\end{array}$ & 156 & 47 & 26 & 229 \\
\hline Direct & $\mathrm{N} / \mathrm{A}$ & $213(84.2)$ & $75(74.3)$ & $288(81.4)$ \\
\hline Indirect & $\mathrm{N} / \mathrm{A}$ & $40(15.8)$ & $26(25.7)$ & $66(18.6)$ \\
\hline \multicolumn{5}{|l|}{ Hospital arrival mode N (\%) } \\
\hline Ambulance & $163(81.5)$ & $206(80.5)$ & $75(74.3)$ & 444 (79.7) \\
\hline Helicopter & $6(3.0)$ & 0 & 0 & $6(1.1)$ \\
\hline Police vehicle & $1(0.5)$ & 0 & 0 & $1(0.2)$ \\
\hline Private vehicle & $30(15.0)$ & $50(19.5)$ & $26(25.7)$ & $106(19.0)$ \\
\hline $\begin{array}{l}\text { Unknown or not documented N } \\
\text { Trauma team activation N (\%) }\end{array}$ & 15 & 0 & 0 & 15 \\
\hline Yes & $20(9.4)$ & $52(21.3)$ & $30(85.7)$ & $102(20.7)$ \\
\hline No & $193(90.6)$ & $192(78.7)$ & $5(14.3)$ & $390(79.3)$ \\
\hline Unknown or not documented N & 2 & 12 & 66 & 80 \\
\hline \multicolumn{5}{|l|}{$\begin{array}{c}\text { Hospital physiological assessment } \\
\text { Mean }( \pm \mathrm{SD})\end{array}$} \\
\hline First BP mmHg & $123.7(21.9)$ & $128.7(21.5)$ & $124.4(30.9)$ & $126.1(23.7)$ \\
\hline Unknown or not documented $\mathrm{N}$ & 10 & 7 & 1 & 18 \\
\hline First pulse rate per min & $91.7(17.6)$ & $\mathrm{N} / \mathrm{A}$ & $89.4(22.9)$ & $90.9(19.5)$ \\
\hline Unknown or not documented $\mathrm{N}$ & 10 & $\mathrm{~N} / \mathrm{A}$ & 1 & 11 \\
\hline Respiration rate per min & $20.3(8.0)$ & $\mathrm{N} / \mathrm{A}$ & $19.5(4.9)$ & $20.0(7.1)$ \\
\hline Unknown or not documented $\mathrm{N}$ & 12 & $\mathrm{~N} / \mathrm{A}$ & 1 & 13 \\
\hline Blood PH & $7.38(0.1)$ & $\mathrm{N} / \mathrm{A}$ & $\mathrm{N} / \mathrm{A}$ & $7.38(0.1)$ \\
\hline
\end{tabular}


Table 3. Cont.

\begin{tabular}{|c|c|c|c|c|}
\hline Population Descriptor & KSMC & BCH & YGH & Total \\
\hline $\begin{array}{c}\text { Unknown or not documented N } \\
\text { Respiration assistance N (\%) }\end{array}$ & 48 & $\mathrm{~N} / \mathrm{A}$ & $\mathrm{N} / \mathrm{A}$ & 48 \\
\hline Yes & $76(39.4)$ & $\mathrm{N} / \mathrm{A}$ & $\mathrm{N} / \mathrm{A}$ & $76(39.4)$ \\
\hline No & $117(60.6)$ & $\mathrm{N} / \mathrm{A}$ & $\mathrm{N} / \mathrm{A}$ & $117(60.6)$ \\
\hline $\begin{array}{c}\text { Unknown or not documented N } \\
\text { GCS }\end{array}$ & 22 & $\mathrm{~N} / \mathrm{A}$ & $\mathrm{N} / \mathrm{A}$ & 22 \\
\hline Mean $( \pm S D)$ & $13.8(3.2)$ & $14.3(2.1)$ & $13.5(3.4)$ & $14.0(2.8)$ \\
\hline \multicolumn{5}{|l|}{ ISS N $(\%)$} \\
\hline$<13$ & $142(66)$ & $174(68.5)$ & $51(50.5)$ & $367(64.4)$ \\
\hline $13-15$ & $22(10.2)$ & $20(7.9)$ & $13(12.9)$ & $55(9.6)$ \\
\hline $16-19$ & $16(7.4)$ & $31(12.2)$ & $18(17.8)$ & $65(11.4)$ \\
\hline $20-28$ & $21(9.8)$ & $12(4.7)$ & $1(1.0)$ & $34(6.0)$ \\
\hline$>28$ & $14(6.5)$ & $17(6.7)$ & $18(17.8)$ & $49(8.6)$ \\
\hline \multicolumn{5}{|l|}{$\begin{array}{c}\text { Injury type N (\%) } \\
\text { Head }\end{array}$} \\
\hline Yes & $56(26.0)$ & $58(22.7)$ & $51(50.5)$ & $165(28.8)$ \\
\hline No & $159(74.0)$ & $198(77.3)$ & $50(49.5)$ & $407(71.2)$ \\
\hline \multicolumn{5}{|l|}{ Spinal } \\
\hline Yes & $74(34.4)$ & $5(2.0)$ & $9(8.9)$ & $88(15.4)$ \\
\hline No & $141(65.6)$ & $251(98.0)$ & $92(91.1)$ & $484(84.6)$ \\
\hline \multicolumn{5}{|l|}{ Thorax and abdominal } \\
\hline Yes & $62(28.8)$ & $30(11.7)$ & $30(29.7)$ & $122(21.3)$ \\
\hline No & $153(71.2)$ & $226(88.3)$ & $71(70.3)$ & $450(78.7)$ \\
\hline \multicolumn{5}{|l|}{ Other type of injury } \\
\hline Yes & $175(81.4)$ & $214(83.6)$ & $67(66.3)$ & $456(79.7)$ \\
\hline No & $40(18.6)$ & $42(16.4)$ & $34(33.7)$ & $116(20.3)$ \\
\hline Unknown or not documented N & 0 & 0 & 0 & 0 \\
\hline
\end{tabular}

Table 4. Thirty-day patient outcome following a traffic crash.

\begin{tabular}{|c|c|c|c|c|}
\hline Population Descriptor & KSMC & BCH & YGH & Total \\
\hline Total study participants N (\%) & $215(37.8)$ & $256(44.6)$ & $101(17.6)$ & $572(100)$ \\
\hline \multicolumn{5}{|l|}{ Discharge ED N (\%) } \\
\hline Discharged home & $1(0.5)$ & $\mathrm{N} / \mathrm{A}$ & $\mathrm{N} / \mathrm{A}$ & $1(0.5)$ \\
\hline Ward & $177(82.3)$ & $\mathrm{N} / \mathrm{A}$ & $\mathrm{N} / \mathrm{A}$ & $177(82.3)$ \\
\hline ICU or HDU & $24(11.2)$ & $\mathrm{N} / \mathrm{A}$ & $\mathrm{N} / \mathrm{A}$ & $24(11.2)$ \\
\hline Operating theatre & $12(5.6)$ & $\mathrm{N} / \mathrm{A}$ & $\mathrm{N} / \mathrm{A}$ & $12(5.6)$ \\
\hline Other & $1(0.5)$ & $\mathrm{N} / \mathrm{A}$ & $\mathrm{N} / \mathrm{A}$ & $1(0.5)$ \\
\hline \multicolumn{4}{|l|}{ Days in ICU (in days) } & 0 \\
\hline Mean $( \pm \mathrm{SD})$ & $1.5(5.5)$ & $0.2(1.6)$ & $1.5(5.3)$ & $0.9(4.2)$ \\
\hline $\begin{array}{l}\text { Unknown or not documented } \mathrm{N} \\
\text { Days in hospital (in days) }\end{array}$ & 0 & 1 & 0 & 1 \\
\hline Mean $( \pm \mathrm{SD})$ & $13.2(13.4)$ & $2.5(4.1)$ & $4.8(7.5)$ & $7.0(10.4)$ \\
\hline $\begin{array}{l}\text { Unknown or not documented N } \\
\text { Outcome (30 days) N (\%) }\end{array}$ & 0 & 2 & 0 & 2 \\
\hline In healthcare facility & $19(8.8)$ & $4(1.6)$ & $18(17.8)$ & $41(7.2)$ \\
\hline Discharge home & $187(87.0)$ & $228(89.1)$ & $73(72.3)$ & $488(85.3)$ \\
\hline Mortuary/died & $9(4.2)$ & $24(9.4)$ & $10(9.9)$ & $43(7.5)$ \\
\hline Unknown or not documented $\mathrm{N}$ & 0 & 0 & 0 & 0 \\
\hline
\end{tabular}


Due to the amount of missing data, four variables were excluded from the logistic regression analysis. In particular, despite previous literature reporting a correlation between some of these variables and mortality, a number of key variables were not able to be included, such as patient obesity status [23,24], not wearing a seat belt $[25,26]$, and not using a safety helmet $[27,28]$. Age, gender, mechanism of injury, ICU and hospital length of stay, and 30-day mortality were documented comprehensively across all the three sites, with limited missing data.

While data were available in patients' files in two of the hospitals, nurses had to access multiple programs to gather it, which was time-consuming when collecting large data sets. Training staff to collect data (e.g., including the Abbreviated Injury Score (AIS)) was identified as a need. The lack of training on how to collect the AIS was expected [13], and online sources and instructions were provided to support the hospital staff involved in data collection. The average length of the self-training was approximately 4-6 h. How long it took to manually calculate the ISS per person is not known.

Table 5 presents the results of the analyses of patient and hospital characteristics in relation to unadjusted in-hospital, 30-day mortality. The proportion of deaths where head injuries were present was $22.4 \%$ compared to deaths without a head injury $1.5 \%$ $(\mathrm{OR}=19.31,95 \%$ CI: 7.97-46.82, $p<0.001)$. Patients achieving a lower education level (high school or below) had a higher mortality rate (15.2\%) compared to patients who had attained a tertiary education $(6.1 \%)(\mathrm{OR}=2.77,95 \% \mathrm{CI}: 1.14-6.73, p=0.02)$. Unadjusted thirty-day mortality was linked to the presence of thorax/abdominal injury $(p<0.001)$, prehospital and hospital SBP $\leq 90 \mathrm{mmHg}(p<0.001)$, GCS $\leq 8(p<0.001)$, and ISS $\geq 20$ $(p<0.001)$. During the study period, patients in non-trauma-based system hospitals had a higher mortality rate $9.5 \%(\mathrm{OR}=2.40,95 \% \mathrm{CI}: 1.13-5.12, p=0.01)$ compared to $4.2 \%$ in the trauma-based system hospital.

Table 5. Unadjusted risk ratios of road traffic mortality by patients and hospital characteristics.

\begin{tabular}{|c|c|c|c|c|c|}
\hline \multirow[b]{2}{*}{ Variables } & \multirow[b]{2}{*}{$\begin{array}{l}\text { Population } \\
\quad \text { N (\%) }\end{array}$} & \multicolumn{2}{|c|}{ Patient 30-Day Outcome } & \multirow[b]{2}{*}{$\begin{array}{l}\text { Crude OR, } \\
\text { (95\% CI) }\end{array}$} & \multirow[b]{2}{*}{$p$ Value } \\
\hline & & $\begin{array}{l}\text { Died } \\
\text { N (\%) }\end{array}$ & $\begin{array}{l}\text { Survived } \\
\text { N (\%) }\end{array}$ & & \\
\hline Hospital level & & & & $\begin{array}{c}2.40 \\
(1.13-5.12)\end{array}$ & 0.01 \\
\hline Trauma center & $215(37.6)$ & $9(4.2)$ & $206(95.8)$ & & \\
\hline Non-trauma center & $357(62.4)$ & $34(9.5)$ & $323(90.5)$ & & \\
\hline Age group (in years) & & & & $\begin{array}{c}0.74 \\
(0.28-1.98)\end{array}$ & 0.55 \\
\hline $\begin{array}{l}18-54 \\
\geq 55\end{array}$ & $\begin{array}{c}517(90.9) \\
52(9.1)\end{array}$ & $\begin{array}{c}38(7.4) \\
5(9.6)\end{array}$ & $\begin{array}{l}479(92.6) \\
47(90.4)\end{array}$ & & \\
\hline Gender & & & & $\begin{array}{c}0.77 \\
(0.31-1.91)\end{array}$ & 0.57 \\
\hline $\begin{array}{l}\text { Female } \\
\text { Male }\end{array}$ & $\begin{array}{c}63(11.4) \\
491(88.6)\end{array}$ & $\begin{array}{c}6(9.5) \\
37(7.5)\end{array}$ & $\begin{array}{c}57(90.5) \\
452(92.5)\end{array}$ & & \\
\hline Marital status & & & & $\begin{array}{c}1.05 \\
(0.47-2.31)\end{array}$ & 0.90 \\
\hline Married & $204(59.6)$ & $17(8.3)$ & $187(91.7)$ & & \\
\hline $\begin{array}{c}\text { Unmarried } \\
\text { Employment status }\end{array}$ & $138(40.4)$ & $11(8.0)$ & & $\begin{array}{c}0.97 \\
(0.44-2.16)\end{array}$ & 0.95 \\
\hline $\begin{array}{l}\text { Employed } \\
\text { Unemployed }\end{array}$ & $\begin{array}{l}225(67.2) \\
110(32.8)\end{array}$ & $\begin{array}{l}20(8.9) \\
10(9.1)\end{array}$ & $\begin{array}{l}205(91.1) \\
100(90.1)\end{array}$ & & \\
\hline Education level & & & & $\begin{array}{c}2.77 \\
(1.14-6.73)\end{array}$ & 0.02 \\
\hline$\leq$ High school & $79(32.4)$ & $12(15.2)$ & $67(84.8)$ & & \\
\hline Tertiary & $165(67.6)$ & $10(6.1)$ & 155 (93.9) & & \\
\hline
\end{tabular}


Table 5. Cont.

\begin{tabular}{|c|c|c|c|c|c|}
\hline \multirow[b]{2}{*}{ Variables } & \multirow{2}{*}{$\begin{array}{l}\text { Population } \\
\text { N (\%) }\end{array}$} & \multicolumn{2}{|c|}{ Patient 30-Day Outcome } & \multirow{2}{*}{$\begin{array}{c}\text { Crude OR, } \\
\text { (95\% CI) }\end{array}$} & \multirow[b]{2}{*}{$p$ Value } \\
\hline & & $\begin{array}{c}\text { Died } \\
\text { N (\%) }\end{array}$ & $\begin{array}{c}\text { Survived } \\
\text { N (\%) }\end{array}$ & & \\
\hline Injury Time & & & & $\begin{array}{c}0.97 \\
(0.52-1.83)\end{array}$ & 0.94 \\
\hline AM & $317(56.3)$ & $24(7.6)$ & $293(92.4)$ & & \\
\hline PM & $246(43.7)$ & $19(7.7)$ & $227(92.3)$ & & \\
\hline Mechanism of injury & & & & $\begin{array}{c}1.97 \\
(0.99-3.92)\end{array}$ & 0.05 \\
\hline MV-Driver & $332(57.9)$ & $31(9.4)$ & $301(90.6)$ & & \\
\hline Other Mechanism & $240(42.1)$ & $12(5.0)$ & $228(95.0)$ & & \\
\hline Collision type & & & & $\begin{array}{c}10.35 \\
(3.97-26.97)\end{array}$ & $<0.001$ \\
\hline Multiple & $34(14.9)$ & $15(44.1)$ & $19(55.9)$ & & \\
\hline $\begin{array}{c}\text { Other type } \\
\text { Driver liability }\end{array}$ & $137(85.1)$ & $9(7.1)$ & $118(92.9)$ & & \\
\hline Speed & & & & $\begin{array}{c}1.77 \\
(0.22-14.09)\end{array}$ & 0.58 \\
\hline Yes & $217(93.1)$ & $23(10.6)$ & $194(89.4)$ & & \\
\hline No & $16(6.9)$ & $1(6.3)$ & $15(93.8)$ & & \\
\hline Fatigue related & & & & $\begin{array}{c}0.05 \\
(0.01-0.19)\end{array}$ & $<0.001$ \\
\hline Yes & $93(85.3)$ & $8(8.5)$ & $86(91.5)$ & & \\
\hline No & $16(14.7)$ & $10(62.5)$ & $8(8.5)$ & & \\
\hline Distracted/inattentive & & & & $\begin{array}{c}2.99 \\
(0.66-13.55)\end{array}$ & 0.13 \\
\hline Yes & $125(75.8)$ & 17 (13.6) & $108(86.4)$ & & \\
\hline No & $40(24.2)$ & $2(5.0)$ & $38(95.0)$ & & \\
\hline Hospital arrival mode & & & & $\begin{array}{c}0.19 \\
(0.04-0.82)\end{array}$ & 0.01 \\
\hline Ambulance & $450(80.8)$ & $40(8.9)$ & $410(91.1)$ & & \\
\hline $\begin{array}{l}\text { Private/police vehicle } \\
\text { Prehospital procedure- }\end{array}$ & $107(19.2)$ & $2(1.9)$ & $105(98.1)$ & & \\
\hline Naso/oro airway/ETT & & & & $\begin{array}{c}0.22 \\
(0.09-0.52)\end{array}$ & $<0.001$ \\
\hline Yes & $39(11.4)$ & $10(25.6)$ & $29(74.4)$ & & \\
\hline No & $304(88.6)$ & $22(7.2)$ & $282(92.8)$ & & \\
\hline Trauma team activation & & & & $\begin{array}{c}0.06 \\
(0.02-0.14)\end{array}$ & $<0.001$ \\
\hline Yes & $102(20.7)$ & $26(25.5)$ & $76(74.5)$ & & \\
\hline No & $390(79.3)$ & $8(2.1)$ & $382(97.9)$ & & \\
\hline First prehospital SBP & & & & $\begin{array}{c}27.2 \\
(7.98-92.67)\end{array}$ & $<0.001$ \\
\hline$\leq 90 \mathrm{mmHg}$ & $13(4.3)$ & $8(61.5)$ & $5(38.5)$ & & \\
\hline$>90 \mathrm{mmHg}$ & $288(95.7)$ & $16(5.6)$ & $272(94.4)$ & & \\
\hline First hospital SBP & & & & $\begin{array}{c}41.47 \\
(16.70-102.99)\end{array}$ & $<0.001$ \\
\hline$\leq 90 \mathrm{mmHg}$ & $26(4.7)$ & $17(65.4)$ & $9(34.6)$ & & \\
\hline$>90 \mathrm{mmHg}$ & $528(95.3)$ & $23(4.4)$ & $505(95.6$ & & \\
\hline GCS & & & & $\begin{array}{c}123 \\
(48.33-313)\end{array}$ & $<0.001$ \\
\hline$\leq 8$ & $40(7.7)$ & $29(72.5)$ & $11(27.5)$ & & \\
\hline$>8$ & $477(92.3)$ & $10(2.1)$ & $467(97.9)$ & & \\
\hline ISS & & & & $\begin{array}{c}497 \\
(66.79-3710)\end{array}$ & $<0.001$ \\
\hline$<20$ & $487(85.4)$ & $1(0.2)$ & $486(99.8)$ & & \\
\hline$\geq 20$ & $83(14.6)$ & $42(50.6)$ & $41(49.4)$ & & \\
\hline
\end{tabular}


Table 5. Cont.

\begin{tabular}{|c|c|c|c|c|c|}
\hline \multirow[b]{2}{*}{ Variables } & \multirow[b]{2}{*}{$\begin{array}{l}\text { Population } \\
\text { N (\%) }\end{array}$} & \multicolumn{2}{|c|}{ Patient 30-Day Outcome } & \multirow[b]{2}{*}{$\begin{array}{c}\text { Crude OR, } \\
(95 \% \mathrm{CI})\end{array}$} & \multirow[b]{2}{*}{$p$ Value } \\
\hline & & $\begin{array}{l}\text { Died } \\
\text { N (\%) }\end{array}$ & $\begin{array}{c}\text { Survived } \\
\mathbf{N}(\%)\end{array}$ & & \\
\hline Admitted to ICU & & & & $\begin{array}{c}9.16 \\
(4.59-18.29)\end{array}$ & $<0.001$ \\
\hline Yes & $58(10.2)$ & $18(31.0)$ & $40(69.0)$ & & \\
\hline No & $513(89.8)$ & $24(4.7)$ & $489(95.3)$ & & \\
\hline Head injury & & & & $\begin{array}{c}19.31 \\
(7.97-46.82)\end{array}$ & $<0.001$ \\
\hline Yes & $165(28.8)$ & $37(22.4)$ & $128(77.6)$ & & \\
\hline No & $407(71.2)$ & $6(1.5)$ & $401(98.5)$ & & \\
\hline Spinal injury & & & & $\begin{array}{c}0.70 \\
(0.27-1.84)\end{array}$ & 0.47 \\
\hline Yes & $88(15.4)$ & $5(5.7)$ & $83(94.3)$ & & \\
\hline No & $484(84.6)$ & $38(7.9)$ & $446(92.1)$ & & \\
\hline $\begin{array}{c}\text { Thorax/abdominal } \\
\text { injury }\end{array}$ & & & & $\begin{array}{c}4.04 \\
(2.14-7.64)\end{array}$ & \\
\hline Yes & $122(21.3)$ & $21(17.2)$ & $101(82.8)$ & & $<0.001$ \\
\hline No & $450(78.7)$ & $22(4.9)$ & $428(95.1)$ & & \\
\hline
\end{tabular}

Note: in this table, we have included only variables with sufficient data. MV = Motor Vehicle; Oro Airway = Oropharyngeal Airway; ETT = Endotracheal Tube; GCS = Glasgow Coma Scale; ISS = Injury Severity Score. $p$ values derived from chi-square test or Fisher exact test ( $p$ value $<0.05$ was considered significant).

The logistic regression model contained eight variables that contributed significantly to predicting in-hospital, 30-day mortality $\left.\chi^{2}(8, \mathrm{~N}=505)=192.09, p<0.001\right)$. The model correctly classified $92.9 \%$ of cases $(\mathrm{R} 2=0.78)$. The logistic regression model included four independently statistically significant predictors of mortality following an RTC: being treated in a non-trauma system hospital (OR $=6.27, p=0.01)$, hospital SBP $\leq 90 \mathrm{mmHg}$ $(\mathrm{OR}=8.13, p=0.03), \mathrm{GCS}$ score $\leq 8(\mathrm{OR}=22.52, p<0.001)$, and $\mathrm{ISS} \geq 20(\mathrm{OR}=122, p<0.001)$ (Table 6). When severity of injury (indicated by ISS) was controlled, in addition to the above four variables, the model also revealed that sustaining a head injury $(\mathrm{OR}=4.07, p=0.05)$ was an independent, statistically significant predictor of mortality following an RTC.

Table 6. Logistic regression predicting likelihood of mortality following RTCs.

\begin{tabular}{ccc}
\hline Variables & $\begin{array}{c}\text { Adjusted OR, (95\% CI) } \\
\text { Uncontrolled ISS }\end{array}$ & $p$ Value \\
\hline Hospital level: None-TC & $6.27(1.36-28.83)$ & 0.01 \\
Mechanism of injury: MV-Driver & $2.91(0.66-12.81)$ & 0.15 \\
Hospital SBP $\leq$ 90 mmHg & $8.13(1.14-57.78)$ & 0.03 \\
Head injury & $2.71(0.45-16.32)$ & 0.27 \\
Thorax and abdominal injury & $1.61(0.35-7.30)$ & 0.53 \\
Admitted to ICU & $0.29(0.05-1.64)$ & 0.16 \\
GCS $\leq 8$ & $22.52(4.09-123)$ & $<0.001$ \\
ISS $\geq 20$ & $122(12.62-1188)$ & $<0.001$ \\
\hline
\end{tabular}

Note: TC = Trauma Center; GCS = Glasgow Coma Scale; ISS = Injury Severity Score. A $p$ value of $<0.05$ was considered significant.

\section{Discussion}

This study sought to determine the feasibility of sourcing data including sociodemographic variables, crash characteristics, physiological assessment, and prehospital and hospital variables across multiple sites in SA in the absence of regional or national trauma registries. This study also sought to analyze data from three SA hospitals to identify factors associated with RTC patient in-hospital mortality after 30 days. An observational study was conducted appraising the outcomes for RTC patients attending participating hospitals in a three-month period, in which data were extracted from the hospital with a trauma 
registry while manual data collection was required at the two hospitals that do not operate a trauma registry.

The analyses showed significant associations between in-hospital mortality following an RTC and patients being treated in a non-trauma center, having SBP less than or equal to $90 \mathrm{mmHg}$, a GCS score less than or equal to 8 , an ISS equal to or greater than 20 , and sustaining a head injury. These findings are consistent with previous studies $[16,29-35]$. Unadjusted risk ratios of road traffic mortality were further linked to thorax/abdominal injuries $(\mathrm{OR}=4.04)$, being admitted to ICU $(\mathrm{OR}=9.16)$, and crashes with multiple impacts $(\mathrm{OR}=10.35)$. These results are also consistent with earlier research that found that thorax/abdominal injuries [33,34,36], ICU admission [31], and multiple impact collisions [37] were linked to mortality following a crash. Similar to prior research [31], in the current study, the associations between age and gender with mortality were not significant.

Mortality rates were found to be higher for patients who were admitted to a nontrauma center $(\mathrm{OR}=2.40)$. The possible explanations of the higher mortality rate in $\mathrm{BCH}$ and YGH compared to KSMC include the severity of injuries of patients and the challenges facing the emergency medical response systems in these cities. For example, providers of prehospital services in Yanbu city do not have access to advanced life support personnel and equipment. Moreover, as neither hospital is classified as a trauma center, access to the essential components of a trauma service such as specialist-trained personnel, training programs, and quality improvement programs is reduced.

This study further supports the potential benefits of establishing and developing a national trauma system in SA [9]. The introduction of national or regional trauma systems in North American [38,39], European [18,40], Asian [41,42], and Oceanian countries [38,43] has resulted in significant reductions in trauma-related mortality [44]. The difference in the mortality rate between $\mathrm{KSMC}$, a developing trauma center, and the other two hospitals further supports this argument. In our study, trauma team activation occurred in only about one-fifth $(20.7 \%)$ of cases; therefore, implementing efforts to strengthen the connection between prehospital triage and receiving hospitals is recommended.

Assessing the feasibility of the future application of the method utilized in the current study was a primary aim of the research. With respect to the completeness of injury data, approximately half the variables had sufficient data for inclusion in inferential statistical analyses. Variables for which comprehensive data were available included demographic variables such as age and gender, indicators of injury severity such as ISS and GCS, mechanism of injury, injury type, and outcomes such as discharge location and mortality. The amount of missing data for prehospital variables was especially high, which impacted the ability to determine the influence of certain crash characteristics on patient mortality. This indicates that data collected routinely and documented in hospital electronic medical records in SA could be improved by considering the inclusion of more variables.

Furthermore, although some variables such as height and weight were consistently available, there were concerns about data accuracy. This is similar to a previous study that highlighted the issue of the accuracy of patients' medication history data collected at admission in SA [45]. Although previous studies have highlighted the strong links between mortality and whether the driver had used alcohol and/or drugs [21,46-48], obesity [23,24], was not wearing a seat belt $[25,26]$, and not wearing a safety helmet $[27,28]$, in the present study, these variables could not be included in the logistic model due to missing data. It is proposed that a nationwide trauma registry is likely to yield better quality data.

Unlike developed countries such as the U.S., where the Fatality Analysis Reporting System (FARS) was created by the National Highway Traffic Safety Administration [49] to document data regarding fatal injuries [50], in SA, there is no national registry of road crashes routinely documenting data such as patient weight, alcohol and/or drug involvement, mechanism of the crash, and a passenger's location in the car. Implementing a program such as FARS would provide relevant data that could be incorporated into a single comprehensive dataset about fatal RTC injuries. With respect to prehospital data, in 2020, the Saudi Red Crescent Authority (SRCA) that provides this service in SA introduced 
a digital data documentation method which could lead to reliable and accurate prehospital data in the future. A pilot study would be recommended to validate the capacity to link prehospital with hospital data.

Documenting information during care delivery is a fundamental procedure when developing a care plan for patients to optimize health outcomes [51,52]. Implementing consistent and accurate data documentation practices is one of the primary responsibilities of a health professional during care delivery [53,54]. Improvements in data quality could be accomplished by adopting standardized, evidence-based guidelines, providing staff with appropriate training, implementing information technology infrastructure, and providing feedback to staff regarding routinely collected data [55-57]. In SA, there are two sources of road traffic crash data: hospital and police data. Hospital data usually includes relevant information about the patient's medical condition and treatment. Police data usually includes information about the crash itself such as time, place, vehicle type, number of people involved, and main outcome such as death [58]. In SA, the link between these two major sources of data could not be achieved due to the deidentified nature of police data. There remains scope, should refinements to how information about RTC incidents is captured be made, to link hospital and police data to enhance access to crash characteristics for future research endeavors.

In the current study, the time taken to extract data for each patient from the trauma registry was not documented. The time required to screen patients and collect data manually at the other two sites was estimated to be approximately $10 \mathrm{~min}$ per patient. The requirements for multi-site ethics approval can be a significant source of delay in research; however, processes were documented clearly by the participating hospitals, and applications were reviewed in a timely manner. The main ethics approval was obtained from KSMC and $\mathrm{MOH}$; the other two sites requested the research team to provide them with additional approval from the region's General Directorate of Health Affairs, MOH. In addition to a formal application process for accessing data from the trauma registry, the hospital required formal agreement that any manuscripts arising from the registry data would be reviewed prior to submission.

The team had some challenges in matching prospective and retrospective data fields. A review of the literature about the predictors of mortality informed the variables sought in the current study. The registry was able to provide the majority of fields from their minimum dataset. The variables that were missing were either demographic in nature or were characteristics about the crash which were not provided to the hospital, and which could therefore not be included. Given that some of the missing variables have previously been identified as possible mortality predictors following RTCs, opportunities to include additional variables such as patient's weight, marital status, employment status, and education level as data fields in the registry could be considered [59]. Despite this, the benefit of having a trauma registry at KSMC for research in traffic crashes was that it provided standardized, complete data on all relevant RTC cases.

This study provides encouraging results indicating that conducting a multi-center large study to better understand predictors of RTC mortality in SA is feasible, with some acknowledged challenges and a number of key recommendations arising. It is proposed that a large multi-center study could only be conducted with substantial additional, onsite resourcing for, and a commitment to, prospective data capture. Screening a large number of patients in a large multi-center would take a lot of staff time and resourcing. Adding to the resourcing required is the need to provide standardized training across sites. Given that the consistency of a minimum set of injury data collected in all hospitals has clinical and research advantages that extend beyond the current trauma study, any capacity to align and standardize data capture within SA would be beneficial. Extending the trauma registry across all regions in SA would enable the collection of comprehensive standardized data on all RTC cases and eliminate the need for manual screening. Although the lack of training on collecting the AIS was expected, the $4-6 \mathrm{~h}$ of online self-training could be insufficient to be 
proficient at AIS coding. Therefore, strengthening hospitals' resources, such as employing qualified personnel for routine data collection using AIS, is recommended.

This study has several limitations. First, the data reported in this study only represent patients who were admitted to the three selected hospitals. Second, the use of 30-day, in-hospital mortality may have excluded patients who died elsewhere within 30 days, such as at other healthcare facilities or those who died at home post-discharge. However, it is reasonable to assume that patients were only discharged home if acute care was no longer deemed necessary. Third, because the non-trauma registry hospitals $\mathrm{BCH}$ and YGH) provided deidentified data, we cannot determine the characteristics of the small number of patients who did not agree to provide further information that was not available in the hospitals' routinely collected data. However, one of our study aims is to improve the minimum dataset of routine hospital data collection across hospitals in SA, where trauma registries do not exist. Fourth, the lack of sufficient data for some variables limited their inclusion and consideration in our analysis.

\section{Conclusions}

Injuries arising from RTCs are a major health problem globally, with RTCs considered to be the most common mechanism of injury-related deaths in LMICs such as SA. Based on the literature, there are a number of potentially relevant prehospital and hospital factors associated with mortality that were not available from hospital records in this study. Collecting multi-center injury data in SA has logistic challenges, predominantly associated with the comparability and completeness of datasets, as well as the need for manual screening and data collection at some institutions. Being treated in a non-trauma center, and having lower SBP, lower GCS, and higher ISS scores were statistically significant independent predictors of 30-day, in-hospital mortality following an RTC. Having a head injury was also an independent predictor when controlling for injury severity.

Author Contributions: This study was developed by all authors. R.J.A., C.M. and V.L. jointly conceived and designed the study. R.J.A. prepared the first draft of this paper and revised comments from C.M., V.L. and O.O. All authors have read and agreed to the published version of the manuscript.

Funding: This research received no external funding.

Institutional Review Board Statement: This study was approved by the Institutional Review Board Committee at King Saud Medical City, SA (H-01-R-053), the General Department of Research and Studies at the SA Ministry of Health: Buraidah Central Hospital (1440-1249939), Yanbu General Hospital (1440-1398648), and the La Trobe University Human Research Ethics Committee (HEC19095).

Informed Consent Statement: Informed consent was obtained from all subjects involved in the study.

Data Availability Statement: The datasets used during the current study are available from the corresponding author on reasonable request.

Acknowledgments: The authors would like to thank the General Directorate for Research and Studies, Ministry of Health and Saudi TraumA Registry, King Saud Medical City for their support in extracting the data for this study.

Conflicts of Interest: The authors declare no conflict of interest.

\section{References}

1. Alharbi, R.; Mosley, I.; Miller, C.; Hillel, S.; Lewis, V. Factors associated with physical, psychological and functional outcomes in adult trauma patients following road traffic crash: A scoping literature review. Transp. Res. Interdiscip. Perspect. 2019,3 , e100061. [CrossRef]

2. WHO. Global Status Report on Road Safety 2018; World Health Organization: Geneva, Switzerland, 2018.

3. WHO. Global Status Report on Road Safety 2015; World Health Organization: Geneva, Switzerland, 2015.

4. Hofman, K.; Primack, A.; Keusch, G.; Hrynkow, S. Addressing the growing burden of trauma and injury in low-and middleincome countries. Am. J. Public Health 2005, 95, 13-17. [CrossRef] [PubMed]

5. Peden, M.; Scurfield, R.; Sleet, D.; Mohan, D.; Hyder, A.A.; Jarawan, E.; Mathers, C.D. World Report on Road Traffic Injury Prevention; World Health Organization: Geneva, Switzerland, 2004. 
6. Nantulya, V.M.; Reich, M.R. The neglected epidemic: Road traffic injuries in developing countries. BMJ 2002, 324, 1139-1141. [CrossRef]

7. Alanazi, F.; Hussain, S.; Mandil, A.; Alamro, N. Towards an electronic national injury surveillance system in Saudi Arabia. East. Mediterr. Health J. 2015, 21, 140-146. [CrossRef]

8. Traffic Accident Statistics as of 1439 H. Available online: https:/ /www.data.gov.sa/Data/en/dataset/traffic-accident-statisticsas-of-1439-h (accessed on 22 September 2021).

9. Alharbi, R.J.; Lewis, V.; Mosley, I.; Miller, C. Current trauma care system in Saudi Arabia: A scoping literature review. Accid. Anal. Prev. 2020, 144, 105653. [CrossRef] [PubMed]

10. Lindström, V.; Bohm, K.; Kurland, L. Prehospital care in Sweden. Notf. Rett. 2015, 18, 107-109. [CrossRef]

11. Altwaijri, S.; Quddus, M.; Bristow, A. Analysing the severity and frequency of traffic crashes in riyadh city using statistical models. Int. J. Transp. Sci. Technol. 2012, 1, 351-364. [CrossRef]

12. Harrington, D.T.; Connolly, M.; Biffl, W.L.; Majercik, S.D.; Cioffi, W.G. Transfer times to definitive care facilities are too long: A consequence of an immature trauma system. Ann. Surg. 2005, 241, 961-966, discussion 966-8. [CrossRef]

13. Alharbi, R.; Miller, C.; Lewis, V. Protocol for a feasibility exploratory multicentre study of factors influencing trauma patients' outcomes of traffic crashes in Saudi Arabia. BMJ Open 2019, 9, e032046. [CrossRef]

14. GAFS. General Authority for Statistics, Demographic Research Bulletin; GAFS: Riyadh, Saudi Arabia, 2016.

15. Alqahtani, A.S.; Abuzinada, S.A.; Cameron, P.A.; Fitzgerald, M.C.; Alenizi, A.S.; Farjou, D. Experience gained from the implementation of the Saudi TraumA Registry (STAR). BMC Health Serv. Res. 2020, 20, 1-7.

16. Talving, P.; Teixeira, P.G.; Barmparas, G.; DuBose, J.; Preston, C.; Inaba, K.; Demetriades, D. Motorcycle-related injuries: Effect of age on type and severity of injuries and mortality. J. Trauma Acute Care Surg. 2010, 68, 441-446. [CrossRef] [PubMed]

17. Lai, M.-M.; Lin, C.-C.; Lin, C.-C.; Liu, C.-S.; Li, T.-C.; Kao, C.-H. Long-Term Use of Zolpidem Increases the Risk of Major Injury: A Population-Based Cohort Study. In Mayo Clinic Proceedings; Elsevier: Amsterdam, The Netherlands, 2014; pp. 589-594.

18. MacKenzie, E.J.; Rivara, F.P.; Jurkovich, G.J.; Nathens, A.B.; Frey, K.P.; Egleston, B.L.; Salkever, D.S.; Scharfstein, D.O. A national evaluation of the effect of trauma-center care on mortality. N. Engl. J. Med. 2006, 354, 366-378. [CrossRef] [PubMed]

19. Kim, H.-Y. Statistical notes for clinical researchers: Chi-squared test and Fisher's exact test. Restor. Dent. Endod. 2017, 42, 152-155. [CrossRef]

20. Chen, P.-L.; Pai, C.-W. Evaluation of injuries sustained by motorcyclists in approach-turn crashes in Taiwan. Accid. Anal. Prev. 2019, 124, 33-39. [CrossRef]

21. Wiratama, B.S.; Chen, P.-L.; Ma, S.-T.; Chen, Y.-H.; Saleh, W.; Lin, H.-A.; Pai, C.-W. Evaluating the combined effect of alcoholinvolved and un-helmeted riding on motorcyclist fatalities in Taiwan. Accid. Anal. Prev. 2020, 143, 105594. [CrossRef]

22. Chien, D.-K.; Hwang, H.-F.; Lin, M.-R. Injury severity measures for predicting return-to-work after a traumatic brain injury. Accid. Anal. Prev. 2017, 98, 101-107. [CrossRef] [PubMed]

23. Donnelly, J.P.; Griffin, R.L.; Sathiakumar, N.; McGwin, G., Jr. Obesity and vehicle type as risk factors for injury caused by motor vehicle collision. J. Trauma Acute Care Surg. 2014, 76, 1116-1121. [CrossRef]

24. Rice, T.M.; Zhu, M. Driver obesity and the risk of fatal injury during traffic collisions. Emerg. Med. J. 2014, 31, 9-12. [CrossRef]

25. Lardelli-Claret, P.; Espigares-Rodríguez, E.; Amezcua-Prieto, C.; Jiménez-Moleón, J.J.; de Dios Luna-del-Castillo, J.; BuenoCavanillas, A. Association of age, sex and seat belt use with the risk of early death in drivers of passenger cars involved in traffic crashes. Int. J. Epidemiol. 2009, 38, 1128-1134. [CrossRef]

26. Awadzi, K.D.; Classen, S.; Hall, A.; Duncan, R.P.; Garvan, C.W. Predictors of injury among younger and older adults in fatal motor vehicle crashes. Accid. Anal. Prev. 2008, 40, 1804-1810. [CrossRef]

27. Kashani, A.T.; Rabieyan, R.; Besharati, M.M. Modeling the effect of operator and passenger characteristics on the fatality risk of motorcycle crashes. J. Inj. Violence Res. 2016, 8, 35.

28. Eustace, D.; Indupuru, V.K.; Hovey, P. Identification of risk factors associated with motorcycle-related fatalities in Ohio. J. Transp. Eng. 2011, 137, 474-480. [CrossRef]

29. Ishii, W.; Hitosugi, M.; Takeda, A.; Baba, M.; Iizuka, R. Factors influencing vehicle passenger fatality have changed over 10 years: A nationwide hospital-based study. Sci. Rep. 2020, 10, 1-9. [CrossRef] [PubMed]

30. Hamidun, R.; Roslan, A.; Sarani, R. Exploring factors for pedestrian fatalities at junctions in Malaysia. Pertanika J. Soc. Sci. Humanit. 2017, 25, 1833-1840.

31. Saidi, H.; Mutiso, B.K.; Ogengo, J. Mortality after road traffic crashes in a system with limited trauma data capability. J. Trauma Manag. Outcomes 2014, 8, 4. [CrossRef]

32. Koval, K.J.; Cooley, M.; Cantu, R.V.; Sprott, K.F. The effects of alcohol on in-hospital mortality in drivers admitted after motor vehicle accidents. Bull. NYU Hosp. Jt. Dis. 2008, 66, 27-34.

33. Markogiannakis, H.; Sanidas, E.; Messaris, E.; Koutentakis, D.; Alpantaki, K.; Kafetzakis, A.; Tsiftsis, D. Predictors of in-hospital mortality of trauma patients injured in vehicle accidents. Ulus. Travma Acil Cerrahi Derg. 2008, 14, 125-131.

34. Ankarath, S.; Giannoudis, P.V.; Barlow, I.; Bellamy, M.; Matthews, S.; Smith, R. Injury patterns associated with mortality following motorcycle crashes. Injury 2002, 33, 473-477. [CrossRef]

35. Ashley, D.W.; Pracht, E.E.; Medeiros, R.S.; Atkins, E.V.; NeSmith, E.G.; Johns, T.J.; Nicholas, J.M. An analysis of the effectiveness of a state trauma system: Treatment at designated trauma centers is associated with an increased probability of survival. J. Trauma Acute Care Surg. 2015, 78, 706-714. [CrossRef] 
36. Leung, C.; Lui, C.; Tsui, K. Predictors of outcomes of pedestrian casualties in motor vehicle injuries managed in emergency department. Hong Kong J. Emerg. Med. 2013, 20, 82-89. [CrossRef]

37. Ryb, G.E.; Dischinger, P.C.; McGwin, G.; Griffin, R.L. Crash-Related Mortality and Model Year: Are Newer Vehicles Safer? In Annals of Advances in Automotive Medicine/Annual Scientific Conference; Association for the Advancement of Automotive Medicine: Chicago, IL, USA, 2011; p. 113.

38. Demetriades, D.; Martin, M.; Salim, A.; Rhee, P.; Brown, C.; Chan, L. The effect of trauma center designation and trauma volume on outcome in specific severe injuries. Ann. Surg. 2005, 242, 512-517, discussion 517-9. [CrossRef] [PubMed]

39. Moore, L.; Turgeon, A.F.; Lauzier, F.; Emond, M.; Berthelot, S.; Clément, J.; Bourgeois, G.; Lapointe, J. Evolution of patient outcomes over 14 years in a mature, inclusive Canadian trauma system. World J. Surg. 2015, 39, 1397-1405. [CrossRef]

40. Chiara, O.; Mazzali, C.; Lelli, S.; Mariani, A.; Cimbanassi, S. A population based study of hospitalised seriously injured in a region of Northern Italy. World J. Emerg. Surg. 2013, 8, 32. [CrossRef]

41. Leung, K.; Ho, W.; Tong, K.; Yuen, W.K. Outcome of severely injured trauma patients at a designated trauma centre in the Hong Kong Special Administrative Region. Chin. Med. J. 2010, 123, 1251-1254. [PubMed]

42. Siman-Tov, M.; Radomislensky, I.; Peleg, K. Reduction in trauma mortality in Israel during the last decade (2000-2010): The impact of changes in the trauma system. Injury 2013, 44, 1448-1452. [CrossRef] [PubMed]

43. Curtis, K.; Chong, S.; Mitchell, R.; Newcombe, M.; Black, D.; Langcake, M. Outcomes of severely injured adult trauma patients in an Australian health service: Does trauma center level make a difference? World J. Surg. 2011, 35, 2332-2340. [CrossRef] [PubMed]

44. Alharbi, R.J.; Shrestha, S.; Lewis, V.; Miller, C. The effectiveness of trauma care systems at different stages of development in reducing mortality: A systematic review and meta-analysis. World J. Emerg. Surg. 2021, 16, 1-12. [CrossRef]

45. AbuYassin, B.H.; Aljadhey, H.; Al-Sultan, M.; Al-Rashed, S.; Adam, M.; Bates, D.W. Accuracy of the medication history at admission to hospital in Saudi Arabia. Saudi Pharm. J. 2011, 19, 263-267. [CrossRef]

46. Romano, E.; Voas, R.B. Drug and alcohol involvement in four types of fatal crashes. J. Stud. Alcohol Drugs 2011, 72, 567-576. [CrossRef]

47. Siskind, V.; Steinhardt, D.; Sheehan, M.; O'Connor, T.; Hanks, H. Risk factors for fatal crashes in rural Australia. Accid. Anal. Prev. 2011, 43, 1082-1088. [CrossRef]

48. Beirness, D.J.; Gu, K.W.; Lowe, N.J.; Woodall, K.L.; Desrosiers, N.A.; Cahill, B.; Porath, A.J.; Peaire, A. Cannabis, alcohol and other drug findings in fatally injured drivers in Ontario. Traffic Inj. Prev. 2020, 22, 1-6. [CrossRef] [PubMed]

49. NHTSA. Traffic Safety Facts: A Compilation of Motor Vehicle Crash Data from the Fatality Analysis Reporting System and the General Estimates System; U.S. Department of Transportation: Washington, DC, USA, 2010; p. 20590.

50. Briggs, N.C.; Levine, R.S.; Haliburton, W.P.; Schlundt, D.G.; Goldzweig, I.; Warren, R.C. The Fatality Analysis Reporting System as a tool for investigating racial and ethnic determinants of motor vehicle crash fatalities. Accid. Anal. Prev. 2005, 37,641-649. [CrossRef] [PubMed]

51. Ammenwerth, E.; Mansmann, U.; Iller, C.; Eichstädter, R. Factors affecting and affected by user acceptance of computer-based nursing documentation: Results of a two-year study. J. Am. Med. Inform. Assoc. 2003, 10, 69-84. [CrossRef] [PubMed]

52. Björvell, C.; Wredling, R.; Thorell-Ekstrand, I. Prerequisites and consequences of nursing documentation in patient records as perceived by a group of registered nurses. J. Clin. Nurs. 2003, 12, 206-214. [CrossRef]

53. Beeber, A.S.; Palmer, C.; Waldrop, J.; Lynn, M.R.; Jones, C.B. The role of doctor of nursing practice-prepared nurses in practice settings. Nurs. Outlook 2019, 67, 354-364. [CrossRef]

54. Mathioudakis, A.; Rousalova, I.; Gagnat, A.A.; Saad, N.; Hardavella, G. How to keep good clinical records. Breathe 2016, 12, 369-373. [CrossRef]

55. de Lusignan, S.; van Weel, C. The use of routinely collected computer data for research in primary care: Opportunities and challenges. Fam. Pract. 2006, 23, 253-263. [CrossRef]

56. De Lusignan, S.; Wells, S.; Shaw, A.; Rowlands, G.; Crilly, T. A knowledge audit of the managers of primary care organizations: Top priority is how to use routinely collected clinical data for quality improvement. Med. Inform. Internet Med. 2005, 30, 69-80. [CrossRef]

57. De Lusignan, S. An educational intervention, involving feedback of routinely collected computer data, to improve cardiovascular disease management in UK primary care. Methods Inf. Med. 2007, 46, 57-62. [CrossRef]

58. Roudsari, B.S.; Mock, C.N.; Kaufman, R.; Grossman, D.; Henary, B.Y.; Crandall, J. Pedestrian crashes: Higher injury severity and mortality rate for light truck vehicles compared with passenger vehicles. Inj. Prev. 2004, 10, 154-158. [CrossRef]

59. Alharbi, R.J.; Lewis, V.; Miller, C. A state-of-the-art review of factors that predict mortality among traumatic injury patients following a road traffic crash. Australas. Emerg. Care 2021. Online ahead of print. [CrossRef] [PubMed] 